\title{
Existential behavioral therapy for informal caregivers of palliative patients: Barriers and promoters of support utilization
}

\author{
TAMARA THURN, M.A., ${ }^{1}$ MONIKA BRANDSTÄTTER, M.A. ${ }^{1}$ VERONIKA FENSTERER, M.A. ${ }^{2}$ \\ HELMUT KÜCHENHOFF, PH.D., ${ }^{2}$ AND MARTIN JOHANNES FEGG, PH.D. ${ }^{1}$ \\ ${ }^{1}$ Department of Palliative Medicine, Ludwig-Maximilians-University, Munich, Germany \\ ${ }^{2}$ Statistical Consulting Unit, Department of Statistics, Ludwig-Maximilians-University, Munich, Germany \\ (RECEIVed February 6, 2014; ACCEPTEd April 26, 2014)
}

\begin{abstract}
Objective: Several interventions have been developed during recent years to support informal caregivers of palliative patients. However, these trials reported low enrollment rates. Employing a newly developed group intervention, existential behavioral therapy (EBT), one study reported that only $13.6 \%$ of approached informal caregivers participated. The purpose of our present study was to identify the reasons for this low enrollment rate in order to improve future support designs.

Method: All participants in the EBT trial (intervention vs. standard-care control group) as well as those who declined participation during a 4-month recruitment period were studied prospectively over 12 months. Andersen's behavioral model of healthcare service use was employed to identify group differences between acceptors and decliners: predisposing (age, gender, education, family status, relationship), enabling (social support, distance to hospital, caring vs. bereaved), and need factors ( psychological distress, quality of life) were evaluated in a binary-logistic model.

Results: Some 94 decliners were compared to 160 EBT participants ( $n=81$ intervention, $n=79$ control). Caregivers who took part were significantly more distressed and suffered from a lower quality of life compared to decliners. Not only these need factors but also predisposing (age $<55$ years) and enabling (use of social/professional support, familiarity with caregiving institution) factors were associated with EBT utilization. At the 12-month follow-up, EBT intervention participants reported greater quality of life improvements than decliners or controls $(p=0.05)$. While all groups had mean anxiety scores below the cutoff at 12 -month follow-up, decliners showed better improvement in anxiety compared to EBT participants (intervention $p=0.04$, controls $p=0.03$ ).

Significance of results: On average, decliners are less burdened: they may be more resilient, may have better coping strategies, or already have a sufficient support network in place. Screening caregivers with regard to their experienced quality of life and targeting those in need, especially younger caregivers with low levels of quality of life, may help to allocate resources more appropriately.
\end{abstract}

KEYWORDS: Informal caregivers, Palliative care, Utilization, Behavioral model of healthcare service use, Caregiver interventions

Address correspondence and reprint requests to: Martin Fegg, Department of Palliative Medicine, Ludwig-Maximilians-University Munich, Marchioninistr. 15, 81377 Munich, Germany. E-mail: martin@fegg.de. Website: www.meaninginlife.info

\section{BACKGROUND}

Informal caregivers of palliative patients are close friends, relatives, and partners who have a significant relationship with and provide assistance to a patient with a life-threatening, incurable illness 
(Applebaum \& Breitbart, 2013). Informal caregivers experience intense emotional burdens: self-ratings and standardized interviews show that about 30 to $50 \%$ suffer from psychological distress (Pitceathly \& Maguire, 2003). Increased levels of depression and anxiety have also been reported (Chentsova-Dutton et al., 2002; Pitceathly \& Maguire, 2003).

The authors of a Cochrane review observed that only a few interventions for informal caregivers have been developed, and they are often without a solid theoretical background and do not show long-term intervention effects (Candy et al., 2011). A more recent systematic review found that the number of interventions is increasing but that evaluation of their methodology is still lacking (Applebaum \& Breitbart, 2013).

Existential behavioral therapy (EBT) was developed in order to address these issues. It is based on cognitive behavioral and existential psychology. This novel intervention has been evaluated in a randomized controlled trial, where improvements in both quality of life and depressive symptoms were observed up to one year after completion of EBT (Fegg et al., 2013).

Questions about values, mindfulness, and acceptance have been integrated into the "third wave" of cognitive behavioral therapy (Hayes, 2004). In addition to these essential improvements, EBT includes an existential perspective to meet the needs of informal caregivers in their confrontation with death, hopelessness, questions of meaning, and reorientation toward life (Yalom, 1980).

After developing a treatment manual and conducting a pilot study, EBT treatment effects were evaluated in a randomized controlled trial assessing the quality of life and psychological distress of informal caregivers. The intervention comprised six sessions (22 hours total) (Fegg et al., 2013):

- First meeting: becoming acquainted and introduction to mindfulness.

- Second meeting: death, bereavement, and mindfulness.

- Third meeting: activating resources and finding meaning.

- Fourth meeting: self-care and stress managememt.

- Fifth meeting: personal values for (re-)orientation.

- Sixth meeting: saying goodbye and new steps.

The details of this intervention are described elsewhere (Fegg et al., 2013).
Existential behavioral therapy was designed to support relatives continuously through the processes of caring to grieving (Benkel et al., 2009; Field et al., 2007). Because almost half of the patients died during their stay at the palliative care unit (average treatment period: 10 days), the majority of relatives (77.4\%) were already grieving when the intervention began. Comparable to previous studies, the rate of EBT enrollment was low, with only $13.6 \%$ of approached relatives taking part (range of enrollment in the literature: 2.4-30\%) (Cherlin et al., 2007; Harding et al., 2004; Bergman et al., 2010).

This highlights the fact that further studies are needed to investigate the factors that influence utilization of mental health prevention and treatment programs in palliative care (Wills \& Gibbons, 2009). Their findings may provide ideas for better design and implementation of interventions that would lead to improved acceptance (Harding et al., 2004).

There are two main perspectives in research on mental healthcare service utilization to explain help seeking and to analyze the "complex array of psychological, social, and demographic factors" influencing a distressed individual's decision to utilize services (Henshaw \& Freedman-Doan, 2009, p. 420).

The health belief model (Rosenstock, 1966), a sociocognitive approach, explains preventive health behavior as a result of a rational decision-making process. This model proposes two groups of variables influencing utilization of healthcare services: (1) readiness to act and (2) belief regarding the relative beneficial value of an available action/intervention. Readiness to act is defined by an individual's perceived vulnerability to a particular health problem and the perceived seriousness of this problem. Beliefs regarding the relative beneficial value of utilizing healthcare services are based on a cost/benefit analysis. The model proposes that people are most likely to utilize healthcare services when they: (1) believe they are at high risk ( perceived susceptibility), (2) believe that it is a serious problem with severe consequences (perceived seriousness), (3) believe that the intervention will be effective (perceived benefits), and (4) perceive few barriers to service utilization (perceived barriers). All variables are thought to be influenced by such sociodemographic characteristics as age, gender, and education (Henshaw \& Freedman-Doan, 2009).

Andersen's behavioral model of healthcare service utilization, based on a structural approach, was developed to analyze factors associated with utilization of healthcare services (including inpatient care, physician visits, and psychological support) (Andersen, 1995; deBoer et al., 1997; Phillips et al., 1998). According to this conceptual model, use is determined by individual characteristics, environmental influences, 
and the interaction between internal and external factors (Andersen, 1995). The model postulates three factors representing individual determinants of utilization:

Predisposing factors (e.g., age, gender, race, health beliefs, sociocultural environment) define a person's willingness to seek help. Female gender, younger age, and higher education, for example, seem to be associated with a higher level of psychological help seeking (Toseland et al., 2002; Cherlin et al., 2007; Dhingra et al., 2010).

Enabling factors (e.g., family support, social support, access to health insurance, one's community) describe aspects that may facilitate access to services. For example, informal support from one's social network or familiarity with professional support systems may enhance the likelihood of utilizing psychosocial support (Toseland et al., 2002).

Need factors (individually perceived and actual need for healthcare service): higher distress or impairments are often associated with higher levels of service use (deBoer et al., 1997; Cherlin et al., 2007; Bergman et al., 2010; 2011). Need variables are considered to be the most immediate determinants for utilization of support (Andersen, 1995; deBoer et al., 1997; Bergman et al., 2010).

We chose Andersen's model as a theoretical framework for our study due to several reasons. First, the behavioral model of healthcare service use is more commonly applied in a wide range of research (Andersen, 2008) and has been employed to explain and predict health behavior and help seeking in bereavement (Bergman et al., 2010; 2011), caregivers for dementia patients (Toseland et al., 2002), chronic illness (deBoer et al., 1997), and treatment of mental problems (Dhingra et al., 2010). Moreover, the behavioral model was specifically developed for analyzing factors associated with utilization of healthcare services and can be used to integrate a wide range of individual as well as contextual variables (e.g., access to informal support sources or provider-related variables) associated with decisions to seek help.

\footnotetext{
Aim

Our study aimed to (1) identify the characteristics of EBT utilization with respect to the behavioral model of healthcare service use, (2) explore individually given reasons for declining support during the EBT trial, and (3) analyze whether participants and decliners differ in their long-term (12-month) psychological distress and quality of life (QoL).
}

\section{METHODS}

\section{Design and Participants}

Our study had a prospective longitudinal design with two assessments (baseline and 12-month follow-up). Inclusion criteria comprised being a relative of a palliative care patient (life expectancy $\leq 6$ months according to the patient's physician), being at least 21 years of age, and having a sufficient knowledge of German. The exclusion criterion was a severe mental illness that would adversely affect the ability to give informed consent (e.g., dementia, delirium, acute psychosis). Two palliative care units and a radiation oncology department in Munich took part. The EBT sessions were held at one palliative care ward. Informal caregivers were approached as soon as possible after their ill relative was admitted to the palliative inpatient facility either by phone or in person to inform them about the EBT trial. They were given written and oral information by a member of our research group on the format (group format, led by a psychotherapist) and duration (six sessions) of the intervention, as well as about the content of each session. They were informed that the intervention was new and being currently evaluated. Caregivers were informed that if they were interested in participating they would be randomly assigned to either the intervention or control group, and in either case would be asked to complete several questionnaires. Care was taken to make this clear from the onset. In addition, help was offered to find alternative support, if desired. During a four-month period (April to June of 2010), all caregivers who declined participation in the EBT trial were asked to take part in the current study. Their data were compared to all relatives who were enrolled in the EBT trial and were randomly assigned to either the intervention or standard-care control group (Fegg et al., 2013). The ethics committee of the Ludwig-Maximilians-University Munich approved our study.

\section{Questionnaires}

Caregivers received a self-administered questionnaire assessing their sociodemographic information, their relationship to the patient, how often they received support (from family, friends, doctors, nurses, psychologists) during the prior four weeks (number of talks to a provider), the time spent providing care per day (in hours), and how intense they rated the pain of the patient (range 0-10). As pain is one of the most distressing physical symptoms at the end of life, we decided to focus on this core physical symptom (Oechsle et al., 2013). Data on patients' age, gender, and time of death were also collected. The 
distance from the home address of the caregiver to the EBT venue was calculated using Google maps.

The Brief Symptom Inventory (BSI) was employed to assess depression, anxiety, and hostility (Derogatis \& Melisaratos, 1983). Previous studies showed that caregivers scored particularly high on these subscales (Chentsova-Dutton et al., 2002). Raw scores were transformed into gender-specific $t$ values $(t \geq$ 60 being clinically relevant). Quality of life was recorded using the Satisfaction with Life Scale (SWLS) (Diener et al., 1985) and a numeric rating scale (QoL-NRS, range $0-10$ on "How is your quality of life at the moment?").

The reasons for declining were collected with open-ended questions and coded a posteriori into six categories (see Table 1). The categories were derived from two studies examining the reasons for not using bereavement services or support groups for caregivers (Cherlin et al., 2007; Gage \& Kinney, 1995).

\section{Statistical Analyses}

Statistical analyses were carried out employing PASW Statistics 18.0 for Windows. The (two-tailed) significance level was set at $\alpha=0.05$.

Student's $t$ and $\chi^{2}$ tests were utilized to examine group differences (RCT participants: intervention and controls vs. decliners). A hierarchical binarylogistic regression based on the behavioral model of healthcare service use was applied to test utilization or decline of support. The predictors were categorized into three groups (predisposing, enabling, and need factors), which were entered consecutively into the analysis. Multiple regression models (adjusted for baseline values, age, gender, relation to the patient, time of death, and use of other support) were applied to examine the long-term effects on psychological distress and QoL in the three groups (EBT, control, and decliners).

Table 1. Reasons for not using EBT $(\mathrm{n}=84)$

\begin{tabular}{lr}
\hline \hline Personal Reasons & $64.3 \%$ \\
Other support available & $29.8 \%$ \\
No support needed & $25.0 \%$ \\
No group setting wanted & $14.3 \%$ \\
Other & $10.7 \%$ \\
Intervention does not fit oun needs & $4.8 \%$ \\
Logistic Barriers & $50.0 \%$ \\
Lack of time & $32.1 \%$ \\
Place/date of session unfeasible & $29.8 \%$ \\
Other & $9.5 \%$ \\
\end{tabular}

Note. Multiple responses were possible.

\section{RESULTS}

\section{Sample Description}

Some 190 caregivers declined to participate in the EBT trial during the study period, while 94 decliners agreed to participate in the utilization study (inclusion rate, $49.5 \%$; see Figure 1); 78 of these caregivers also completed the 12-month follow-up (dropout rate, 17.0\%). The decliners were compared to all EBT trial participants ( $n=160$; intervention: $n=81$, controls: $n=79$ ).

\section{Reasons for Decline}

The reasons for declining are summarized in Table 1.

\section{Differences Between Participants and Decliners at the Time of Decision}

Participating caregivers were about five years younger than decliners; this corresponded to the age of the patient (Table 2). No differences were found regarding gender, education, employment, or relationship to the patient. EBT participants were more likely to be caregivers, and EBT decliners were more likely to be recently bereaved. Caregivers who felt that the patient suffered from greater pain were more likely to participate. No differences were found in terms of time spent providing care per day. A trend was observed indicating that decliners were living further away from the EBT venue compared to participants. Furthermore, relatives of patients being treated at the palliative care ward where the EBT sessions were held were also more likely to participate. EBT participants sought support more often from friends and psychologists during the previous four weeks.

\section{Psychological Distress and Well-Being}

Table 3 shows that EBT participants (intervention and controls) were significantly more distressed (BSI) and reported lower QoL at baseline compared to decliners.

\section{Behavioral Model of Healthcare Service Use}

Figure 2 depicts the results of the binary-logistic regression for utilization predictors, which explain $40.1 \%$ of the overall variance. The regression steps are all significant, indicating that the predictors (predisposing, enabling, need factors) sufficiently discriminate between participants and decliners. The model correctly identifies $75.7 \%$ of caregivers as participants or decliners.

Caregivers who were younger, had a lower QoL, used more sources of social and/or professional support, or whose patient was treated at the palliative 


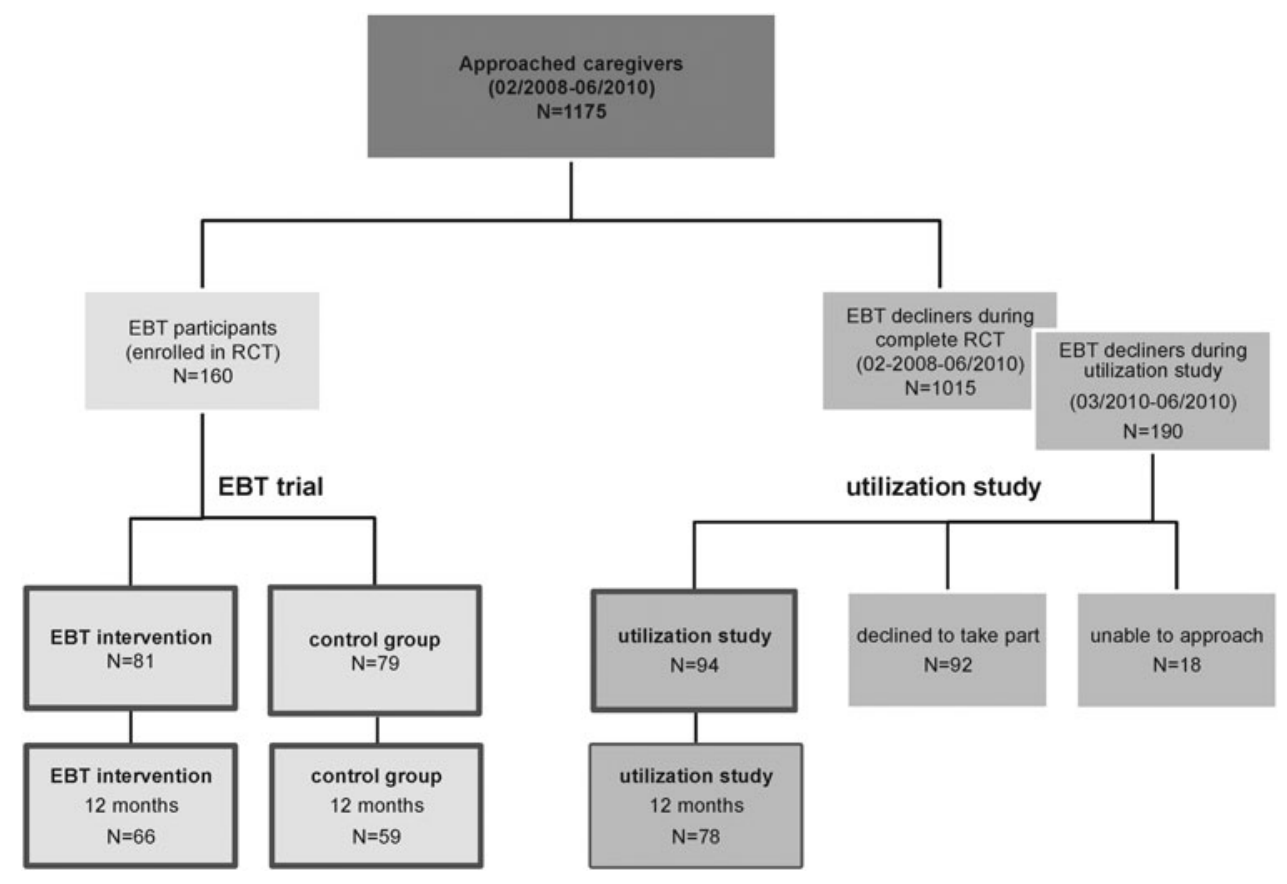

Fig. 1. Flowchart displaying the recruitment process of study participants (decliners and EBT trial participants).

care unit where the EBT sessions were held were more likely to participate in the EBT trial.

\section{Longitudinal Analysis}

Table 3 indicates that decliners were less distressed at baseline than the EBT intervention and control group. Comparing changes between baseline and 12-month follow-up (adjusted for baseline values and other confounders; see Table 4), anxiety was 3.97 and 4.36 points more reduced in decliners (compared to intervention and controls, respectively). With regard to QoL-NRS, the intervention group improved by 0.82 points more than decliners. Decliners showed stronger improvements in anxiety compared to the intervention and control group. We did not find any interaction effects between group (decliners, intervention and control group) and bereavement status.

\section{DISCUSSION}

Despite experiencing intense emotional and psychological burdens, only some informal caregivers of palliative patients access mental healthcare services. This may lead to "chronification" of existing distress (Pitceathly \& Maguire, 2003; Vanderwerker et al., 2005; McNamara \& Rosenwax, 2010). Other factors contributing to chronification may include the experience of additional stressors/life events, multiple losses, or insufficient coping strategies and preexisting psychological disorders. Ambivalence regarding support and time-organizational barriers may also hamper service utilization (Harding \& Higginson, 2001; Payne, 2010).

The behavioral model of healthcare service use provided the framework for identifying barriers and promoters of EBT utilization. Younger age was a predisposing factor, enabling factors included social and professional support already being received as well as familiarity with the caregiving institution, while the need factors were QoL impairments.

Although no significant gender differences were found in utilization behavior, the majority of participants were female. This corresponds with other studies on psychological support utilization (Addis \& Mahalik, 2003; Galdas et al., 2005). Also consistent with previous research, younger caregivers were more likely to participate (Cherlin et al., 2007), while older adults might have more negative attitudes toward seeking help for emotional problems (Segal et al., 2005). Participants were in their mid-fifties, with almost two thirds being the spouse/partner of a palliative care patient. Employment status (including retirement) did not influence these results. Another study offering support groups in the afternoons reported enrollment differences for employed and unemployed caregivers (Harding et al., 2004). EBT sessions, however, were held in the evenings to enable employed caregivers to take part.

EBT groups were conducted at one palliative care department, with informal caregivers being recruited from two other institutions in the same city; familiarity with the venue as well as distance from the caregiver's home were further predictors of EBT utilization. 
Table 2. Sample characteristics of EBT participants $(\mathrm{n}=160)$ and decliners $(\mathrm{n}=94)$

\begin{tabular}{|c|c|c|c|c|}
\hline & EBT Participants & EBT Decliners & Test Statistics & $p$ \\
\hline \multicolumn{5}{|l|}{ Informal Caregivers } \\
\hline Age (years) & $54.4 \pm 13.1$ & $59.3 \pm 13.2$ & $t=2.86$ & 0.01 \\
\hline Gender (female, \%) & $70.3^{--1}$ & 67.0 & $\chi^{2}=0.29$ & 0.59 \\
\hline Education $(\%)$ & & & $\chi^{2}=1.51$ & 0.22 \\
\hline Lower (without graduation, secondary school only) & 54.4 & 62.4 & & \\
\hline Higher (A-levels or university degree) & 45.6 & 37.6 & & \\
\hline Employment (\%) & & & $\chi^{2}=0.08$ & 0.77 \\
\hline Employed (full/part time) & 51.9 & 50.0 & & \\
\hline Unemployed (including homemaker, retired) & 48.1 & 50.0 & & \\
\hline \multicolumn{5}{|l|}{ Social and professional support $(\%)^{\mathrm{a}}$} \\
\hline Family & 82.3 & 78.0 & $\gamma^{2}=0.62$ & 0.43 \\
\hline Friends & 82.3 & 63.4 & $\chi^{2}=10.48$ & 0.01 \\
\hline Doctor & 53.2 & 46.3 & $\chi^{2}=1.01$ & 0.32 \\
\hline Nurse & 29.1 & 23.2 & $\chi^{2}=0.97$ & 0.33 \\
\hline Psychologist & $\mathbf{3 5 . 4}$ & 15.9 & $\chi^{2}=10.11$ & 0.01 \\
\hline Time spent providing care $(\mathrm{hr} /$ day $)$ & $7.3+8.4$ & $7.1+8.4$ & $t=-0.80$ & .94 \\
\hline \multicolumn{5}{|l|}{ Asked to participate in EBT (\%) } \\
\hline Before death of patient & 47.8 & 31.9 & $\chi^{2}=6.08$ & 0.01 \\
\hline After death of patient & 52.2 & 68.1 & & \\
\hline \multicolumn{5}{|l|}{ Caring institution $(\%)^{\mathrm{b}}$} \\
\hline Where EBT sessions were held & 63.3 & 34.0 & $\chi^{2}=20.21$ & $<0.001$ \\
\hline Other institution & 36.7 & 66.0 & & \\
\hline Distance to venue $(\mathrm{km})^{\mathrm{c}}$ & $19.8+23.1$ & $40.5 \pm 102.3$ & $t=1.93$ & 0.06 \\
\hline \multicolumn{5}{|l|}{ Palliative Care Patients } \\
\hline Age & $63.3 \pm 12.5$ & $69.9 \pm 12.9$ & $t=\mathbf{3 . 9 5}$ & $<0.001$ \\
\hline Gender (female) (\%) & $53.2 \div-2$ & $48.9-2-2$ & $\chi^{2}=0.50$ & 0.48 \\
\hline \multicolumn{5}{|l|}{ Patient is ... (\%) } \\
\hline Spouse/partner/child & 63.7 & 59.3 & $\chi^{2}=0.70$ & 0.40 \\
\hline Other (parent, other relative) & 36.3 & 40.7 & & \\
\hline Intensity of pain ${ }^{\mathrm{d}}$ & $6.4 \pm 3.2$ & $4.8 \pm 4.0$ & $t=-2.75$ & 0.01 \\
\hline
\end{tabular}

Note. Bold = significant results.

${ }^{\mathrm{a}} \mathrm{S}$ upport used at least once during the previous four weeks.

${ }^{\mathrm{b}}$ Palliative care department that held the EBT sessions vs. other institutions (another palliative care ward, a radiation oncology department; all in the same city).

'Distance from caregiver's home to EBT venue.

${ }^{\mathrm{d}}$ Caregiver assessment range: 0 (no pain) to 10 (most intense pain).

Table 3. Psychological distress and quality of life of EBT participants $(\mathrm{n}=160)$ and decliners $(\mathrm{n}=94)$ at baseline

\begin{tabular}{|c|c|c|c|c|}
\hline & & $\mathrm{M} \pm S D$ & $t$ & $p$ \\
\hline \multicolumn{5}{|l|}{ Psychological distress } \\
\hline Depression (BSI) & $\begin{array}{l}\text { Participants } \\
\text { Decliners }\end{array}$ & $\begin{array}{r}64.7 \pm 10.0 \\
58.0+12.6\end{array}$ & 4.33 & $<0.001$ \\
\hline Anxiety (BSI) & $\begin{array}{l}\text { Participants } \\
\text { Decliners }\end{array}$ & $\begin{array}{l}59.8 \pm 12.2 \\
56.0 \pm 13.7\end{array}$ & 2.18 & 0.031 \\
\hline Hostility (BSI) & $\begin{array}{l}\text { Participants } \\
\text { Decliners }\end{array}$ & $\begin{array}{l}59.3 \pm 10.5 \\
54.6 \pm 10.8\end{array}$ & 3.31 & 0.001 \\
\hline \multicolumn{5}{|l|}{ Quality of life } \\
\hline Satisfaction with Life (SWLS) & $\begin{array}{l}\text { Participants } \\
\text { Decliners }\end{array}$ & $\begin{array}{l}3.8 \pm 1.3 \\
4.7 \pm 1.3\end{array}$ & -5.06 & $<0.001$ \\
\hline QoL numeric rating scale (QoL-NRS) & $\begin{array}{l}\text { Participants } \\
\text { Decliners }\end{array}$ & $\begin{array}{l}3.7 \pm 2.1 \\
5.6 \pm 2.5\end{array}$ & -6.32 & $<0.001$ \\
\hline
\end{tabular}

Note. Bold $=$ significant results. 


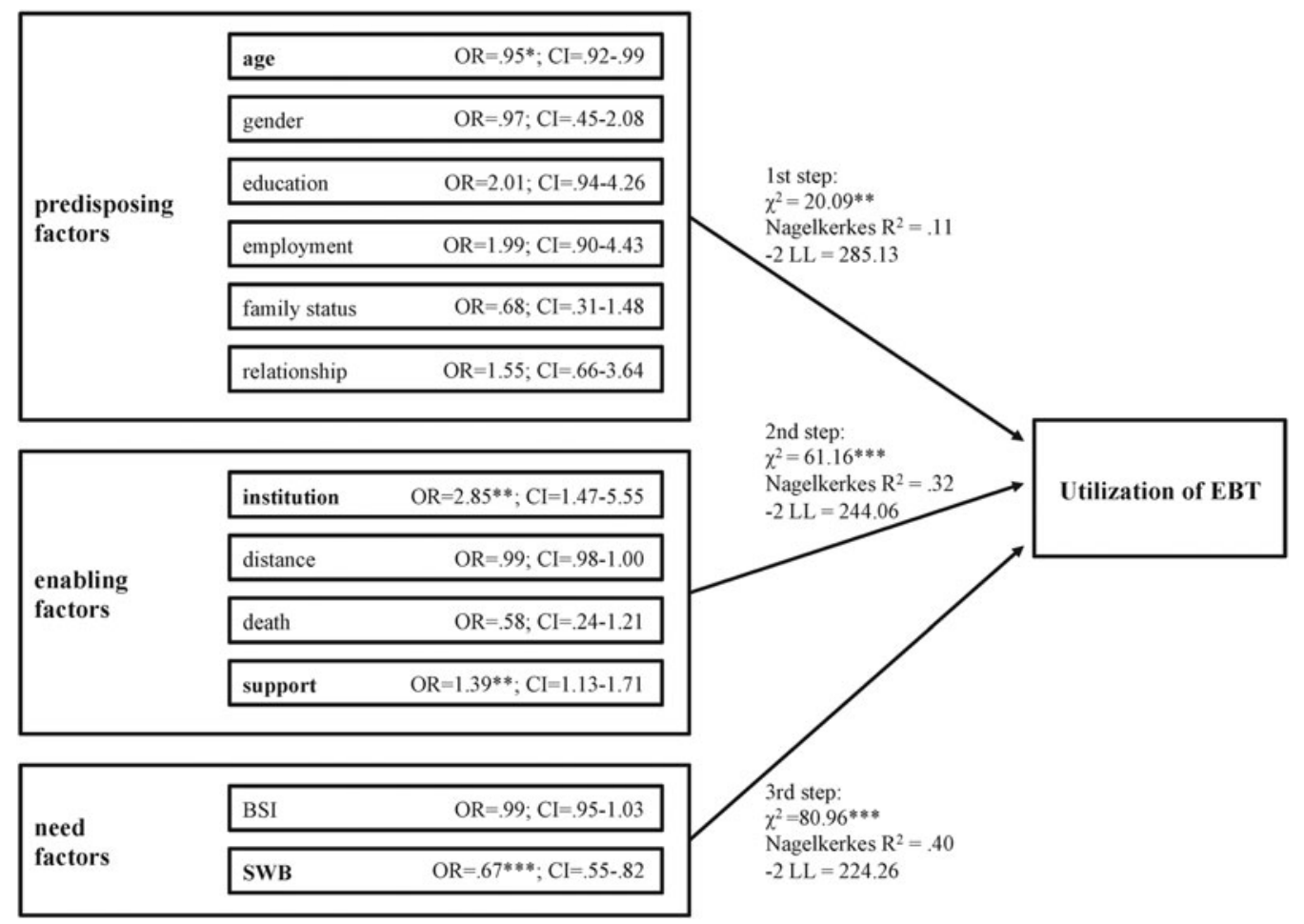

Fig. 2. Behavioral model of healthcare service use: hierarchical binary-logistic regression model. OR $=$ odds ratio; $\mathrm{CI}=$ confidence interval. Outcome: utilization of EBT $(0=$ decliner, $1=$ participant $)$. Predictors: gender $(0=$ female, $1=$ male); education $(0=$ lower level, $1=$ higher level $)$; family status $(0=$ without relationship, $1=$ in a relationship $/ \mathrm{mar}-$ ried); occupation $(0=$ unemployed, $1=$ employed $)$; relation to patient $(0=$ other relation, $1=$ patient is partner $/$ child $)$; institution $(0=$ patient treated at other institution, $1=$ patient treated at EBT venue department); distance $=$ between caregiver's home and EBT venue $(\mathrm{km})$; death $(0=$ patient deceased before EBT recruitment, 1 = patient deceased after EBT recruitment); support $=$ number of sources of social and/or professional support used during the last four weeks; $\mathrm{BSI}=$ psychological distress (composite score of the BSI subscales); SWB = subjective well-being (composite score of QoL-NRS and SWLS). * $p<0.05,{ }^{* *} p<0.01 .{ }^{* * *} p<0.001$.

Compared to decliners, EBT participants (intervention and controls) had significantly higher levels of psychological distress (BSI depression, anxiety, hostility) and significantly lower QoL (SWLS, QoLNRS) at the time of enrollment decision. Due to their increased distress, participants might search for more help from social and professional sources. Seeking help often starts with friends and family; if this turns out to be insufficient, professional support is requested (Barker \& Pistrang, 2001).

At the 12-month follow-up, decliners were again less psychologically distressed and experienced better QoL compared to EBT participants. Changes during the one-year period pointed to the fact that caregivers receiving EBT treatment showed the strongest improvement. In the longitudinal analysis, the intervention group reported higher QoL improvements than decliners at follow-up. Decliners showed stronger improvements in anxiety compared to the intervention and control group. Bereavement status did not influence these anxiety improvements. Though the differences were statistically significant, distress levels for both decliners and EBT intervention participants fell within a normal range (i.e., not pathologically elevated) 12 months later $(t<$ $60)$. Untreated controls, however, still had clinically relevant depressive symptoms. Again, this shows their increased distress and support needs.

In summary, it is notable that caregivers who did not want to utilize EBT were still well adjusted at follow-up. These findings imply that utilization behavior might be a self-selection process in which distressed relatives are more likely to seek support and benefit, particularly from psychological interventions (Kissane et al., 2006; Toseland et al., 2002). It may be helpful to screen informal caregivers for psychological distress and quality-of-life impairments. In addition to the instruments used in our study, the Hospital Anxiety and Depression Scale (Zigmond \& Snaith, 1983) and the Distress Thermometer (Weiss \& Holland, 2007) have been successfully applied in other contexts. Following the screening, psychological support could be targeted at particularly burdened relatives. 
Table 4. Linear regression analyses of long-term changes in psychological distress and quality of life ( $\mathrm{n}=187$ ): EBT participants compared to EBT decliners (intervention vs. decliners // control vs. decliners).

\begin{tabular}{|c|c|c|c|c|c|c|c|}
\hline & \multicolumn{3}{|c|}{$\mathrm{M} \pm S D$} & \multicolumn{4}{|c|}{$C I$} \\
\hline & Baseline & 12 Months & $\Delta_{12 \mathrm{M}-\text { Baseline }}$ & $B$ & Low & High & $p$ \\
\hline \multicolumn{8}{|c|}{ Depression (BSI) } \\
\hline Intervention & $66.0 \pm 9.4$ & $58.9 \pm 10.0$ & $-7.2 \pm 10.2$ & -0.75 & -4.27 & 2.76 & 0.672 \\
\hline Control & $62.6 \pm 10.6$ & $61.5 \pm 10.4$ & $-1.0 \pm 10.8$ & 3.37 & -0.04 & 6.78 & .053 \\
\hline Decliners & $56.2 \pm 11.8$ & $55.0 \pm 11.8$ & $-1.2 \pm 8.5$ & & & & \\
\hline \multicolumn{8}{|l|}{ Anxiety (BSI) } \\
\hline Intervention & $61.1 \pm 12.7$ & $53.7 \pm 11.6$ & $-7.4 \pm 13.2$ & 3.97 & 0.13 & 7.81 & 0.043 \\
\hline Control & $58.3 \pm 10.9$ & $52.8 \pm 11.3$ & $-5.5 \pm 10.6$ & 4.36 & 0.52 & 8.21 & 0.026 \\
\hline Decliners & $55.8 \pm 13.1$ & $49.0 \pm 12.1$ & $-6.8 \pm 11.7$ & & & & \\
\hline \multicolumn{8}{|l|}{ Hostility (BSI) } \\
\hline Intervention & $59.2 \pm 11.5$ & $54.4 \pm 10.5$ & $-4.8 \pm 11.7$ & 1.86 & -1.70 & 5.41 & 0.304 \\
\hline Control & $59.0 \pm 9.1$ & $53.4 \pm 10.5$ & $-5.5 \pm 10.6$ & 1.04 & -2.54 & 4.61 & 0.569 \\
\hline Decliners & $54.3 \pm 10.5$ & $51.4 \pm 11.4$ & $-2.9 \pm 9.6$ & & & & \\
\hline \multicolumn{8}{|l|}{ SWLS } \\
\hline Intervention & $3.8 \pm 1.3$ & $4.4 \pm 1.1$ & $0.5 \pm 1.1$ & 0.15 & -0.25 & 0.56 & 0.449 \\
\hline Control & $4.0 \pm 1.3$ & $4.2 \pm 1.4$ & $0.2 \pm 1.1$ & -0.17 & -0.57 & 0.23 & 0.413 \\
\hline Decliners & $4.8 \pm 1.3$ & $4.9 \pm 1.3$ & $0.1 \pm 1.2$ & & & & \\
\hline \multicolumn{8}{|l|}{ QoL-NRS } \\
\hline Intervention & $3.7 \pm 2.0$ & $6.5 \pm 1.8$ & $2.8 \pm 2.4$ & 0.82 & 0.02 & 1.61 & 0.045 \\
\hline Control & $3.8 \pm 2.3$ & $5.4 \pm 2.4$ & $1.7 \pm 2.5$ & -0.22 & -1.01 & 0.58 & 0.586 \\
\hline Decliners & $5.9 \pm 2.4$ & $6.7 \pm 2.3$ & $0.8 \pm 2.2$ & & & & \\
\hline
\end{tabular}

Note. Bold = significant results. Univariate comparisons between EBT participants (intervention, controls) and decliners controlled for age, gender, relation to patient (partner/child vs. parent/other), time after death of patient (before EBT intervention vs. after EBT intervention).

$B=$ regression coefficient, with positive values indicating higher, negative values indicating lower scores for the EBT participants (intervention vs. controls) compared to EBT decliners.

$C I=95 \%$ confidence interval for $B$. 
Our study has several limitations. Due to logistical barriers, we did not investigate all caregivers who declined to take part during the full length of the EBT trial. Furthermore, the generalizability of our results is limited because only a specific support group was investigated. About 5\% of decliners indicated that the offered intervention did not fit their needs, and about $15 \%$ stated that they did not want to participate in a group setting. Thus, it seems important to offer diverse forms of support, especially both the group and one-on-one support options. At the time of recruitment, a short description of the intervention contents was provided to each caregiver. It is unclear, however, what associations these descriptions elicited in caregivers. This may depend to a large extent on their personal experiences and attitudes. An intervention targeting existential topics potentially attracts a different subgroup of caregivers than an intervention targeting information needs or practical support. Additionally, with six meetings (22 hours in total), EBT is a rather extensive support service compared to intervention doses calculated in a review (mean 7.5 hours; range 1.7-18 hours) (Northouse et al., 2010). More than 30\% of decliners stated that lack of time was one reason they did not use EBT. The enrollment rates for other support services indicate that shorter and/or more flexible interventions (e.g., home visits, phone calls) may be more accessible for relatives of palliative patients, especially for those who provide care for a dying relative (Applebaum \& Breitbart, 2013; Harding et al., 2004). Utilization of EBT may also be confounded by reservations about participating in a randomized controlled trial (i.e., only a $50 \%$ chance to participate, repeated questionnaires), although none of the decliners in this study indicated this as a reason for declining.

Future research could also address the underlying sociocognitive processes (appraisal, attitudes) that affect the decision to utilize or decline support. In addition, attitudinal barriers may inhibit utilization of accessible support despite being required.

To enable distressed caregivers to use support, they need information and knowledge about where to turn for help (Toseland et al., 2002). Thus, palliative care providers should set as a high priority implementing services for caregivers as well as improving the availability and accessibility of support. Familiarity with the service providers is a core promoter of support utilization. Group support may not always be feasible in a palliative care setting. Though cost effective, it might be necessary to initiate support quickly in a one-to-one setting; if the patient is in his or her last days of life, waiting for the start of the next group might be inappropriate. Bereaved caregivers were also less likely to take part in EBT groups, though no negative effects were reported from a "mixed composition" of caring and bereaved relatives (Fegg et al., 2013; Kögler et al., 2013). In summary, interventions for caregivers should be embedded into routine care in hospices and palliative care units at an early stage. Furthermore, early integration of palliative care in treatment of advanced cancer (Greer et al., 2013) may be useful in identifying and targeting distressed patients and their families in need of psychological support services early on.

\section{FUNDING AND CONFLICTS OF INTEREST}

This work was supported by Deutsche Krebshilfe e.V. (No. 107433). The funder had no role in the study design, data collection, analysis or interpretation, decision to publish, or preparation of the manuscript. The authors state that they have no conflicts of interest to declare.

\section{REFERENCES}

Addis, M.E. \& Mahalik, J.R. (2003). Men, masculinity, and the contexts of help seeking. The American Psychologist, $58,5-14$

Andersen, R.M. (1995). Revisiting the behavioral model and access to medical care: Does it matter? Journal of Health and Social Behavior, 36, 1-10.

Andersen, R.M. (2008). National health surveys and the behavioral model of health services use. Medical Care, 46 , $647-653$.

Applebaum, A. \& Breitbart, W. (2013). Care for the cancer caregiver: A systematic review. Palliative \& Supportive Care, 11, 231-252.

Barker, C. \& Pistrang, N. (2001). Psychotherapy and social support: Integrating research on psychological helping. Clinical Psychology Review, 22, 361-379.

Benkel, I., Wijk, H. \& Molander, U. (2009). Family and friends provide most social support for the bereaved. Palliative Medicine, 23, 141-149.

Bergman, E., Haley, W.E. \& Small, B.J. (2010). The role of grief, anxiety and depressive symptoms in the use of bereavement services. Death Studies, 34, 441-458.

Bergman, E.J., Haley, W.E. \& Small, B.J. (2011). Who uses bereavement services? An examination of service use by bereaved dementia caregivers. Aging \& Mental Health, $15,531-540$.

Candy, B., Jones, L., Drake, R., Leurent, B. \& King, M. (2011). Interventions for supporting informal caregivers of patients in the terminal phase of a disease. The Cochrane Database of Systematic Reviews, 6, CD007617. doi: 10.1002/14651858.CD007617.pub2.

Chentsova-Dutton, Y., Shucter, S., Hutchin, S., et al. (2002). Depression and grief reactions in hospice caregivers: From pre-death to 1 year afterwards. Journal of Affective Disorders, 69, 53-60.

Cherlin, E.J., Barry, C.L., Prigerson, H.G., et al. (2007). Bereavement services for family caregivers: How often used, why, and why not. Journal of Palliative Medicine, $10,148-158$. 
deBoer, A., Wijker, W. \& deHaes, H. (1997). Predictors of healthcare utilization in the chronically ill: A review of the literature. Health Policy, 42, 101-115.

Derogatis, L.R. \& Melisaratos, N. (1983). The Brief Symptom Inventory: An introductory report. Psychological Medicine, 13, 595-605.

Dhingra, S.S., Zack, M., Strine, T., et al. (2010). Determining prevalence and correlates of psychiatric treatment with Andersen's behavioral model of health services use. Psychiatric Services, 61, 524-528.

Diener, E., Emmons, R.A., Larsen, R.J., et al. (1985). The Satisfaction With Life Scale. Journal of Personality Assessment, 49, 71-75.

Fegg, M., Brandstätter, M., Kögler, M., et al. (2013). Existential behavioural therapy for informal caregivers of palliative patients: A randomised controlled trial. Psycho-Oncology, 22, 2079-2086.

Field, D., Payne, S., Relf, M., et al. (2007). Some issues in the provision of adult bereavement support by UK hospices. Social Science \& Medicine, 64, 428-438.

Gage, M.J. \& Kinney, J.M. (1995). They aren't for everyone. Clinical Gerontologist, 16, 21-34. Available at http:// www.tandfonline.com/doi/abs/10.1300/J018v16n02_03? journalCode $=$ wcli20preview.

Galdas, P.M., Cheater, F. \& Marshall, P. (2005). Men and health help-seeking behaviour: Literature review. Journal of Advanced Nursing, 49, 616-623.

Greer, J.A., Jackson, V.A., Meier, D.E., et al. (2013). Early integration of palliative care services with standard oncology care for patients with advanced cancer. $C A$ : $A$ Cancer Journal for Clinicians, 63, 349-363.

Harding, R. \& Higginson, I. (2001). Working with ambivalence: Informal carers of patients at the end of life. Supportive Care in Cancer, 9, 642-645.

Harding, R., Higginson, I., Leam, C., et al. (2004). Evaluation of a short-term group of intervention for informal carers of patients attending a home palliative care service. Journal of Pain and Symptom Management, 27, 396-408.

Hayes, S. (2004). Acceptance and commitment therapy, relational frame theory, and the third wave of behavioral and cognitive therapies. Behavior Therapy, 35, 639-665.

Henshaw, E.J. \& Freedman-Doan, C.R. (2009). Conceptualizing mental health care utilization using the health belief model. Clinical Psychology: Science and Practice, 16, $420-444$.

Kissane, D.W., McKenzie, M., Bloch, S., et al. (2006). Family focused grief therapy: A randomized controlled trial in palliative care and bereavement. American Journal of Psychiatry, 163, 1208-1218.

Kögler, M., Brandl, J., Brandstätter, M., et al. (2013). Determinants of the effect of existential behavioral therapy for bereaved partners: A qualitative study. Journal of Palliative Medicine, 16, 1410-1416.

McNamara, B. \& Rosenwax, L. (2010). Which carers of family members at the end of life need more support from health services and why? Social Science \& Medicine, 70, 1035-1041.

Northouse, L.L., Katapodi, M.C., Sing, L., et al. (2010). Intervention with family caregivers of cancer patients. Meta-analysis of randomized trials. CA: A Cancer Journal for Clinicians, 30, 317-339.

Oechsle, K., Goerth, K., Bokemeyer, C., et al. (2013). Symptom burden in palliative care patients: Perspectives of patients, their family caregivers, and their attending physicians. Supportive Care in Cancer, 21, 1955-1962.

Payne, S. (2010). White paper on improving support for family carers in palliative care: part 1: Recommendations from the European Association for Palliative Care (EAPC) Task Force on Family Carers. European Journal of Palliative Care, 17, 238-245.

Phillips, K.A., Morrison, K.R., Andersen, R., et al. (1998). Understanding the context of healthcare utilization: Assessing environmental and provider-related variables in the behavioral model of utilization. Health Service Research, 33, 571-596.

Pitceathly, C. \& Maguire, P. (2003). The psychological impact of cancer on patients' partners and other key relatives: A review. European Journal of Cancer Care, 39, 1517-1524.

Rosenstock, I.M. (1966). Why people use health services. The Milbank Quarterly, 44, 94-124.

Segal, D.L., Coolidge, F.L., Mincic, M.S., et al. (2005). Beliefs about mental illness and willingness to seek help: A crosssectional study. Aging \& Mental Health, 9, 363-367.

Toseland, R.W., McCallion, P., Gerber, T., et al. (2002). Predictors of health and human services use by persons with dementia and their family caregivers. Social Science \& Medicine, 55, 1255-1266.

Vanderwerker, L.C., Laff, R.E., Kadan-Lottick, N.S., et al. (2005). Psychiatric disorders and mental health service use among caregivers of advanced cancer patients. Journal of Clinical Oncology, 23, 6899-6907.

Weiss, T. \& Holland, J. (2007). A single-item rapid screen for distress: Validation of the distress thermometer. Psycho-Oncology, 16, S10-S11.

Wills, T.A. \& Gibbons, F.X. (2009). Commentary: Using psychological theory in help-seeking research. Clinical Psychology: Science and Practice, 16, 440-444.

Yalom, I. (1980). Existential psychotherapy. New York: Basic Books.

Zigmond, A. \& Snaith, R. (1983). The Hospital Anxiety and Depression Scale. Acta Psychiatrica Scandinavica, 67, $361-370$ 